\title{
La cultura como proyecto educativo de una narración compleja
}

Culture as an educational project of a complex narrative

Dr. Salvatore Patera

Universidad del Salento

toto.patera@gmail.com

\section{Resumen}

En una sociedad compleja como la actual, el reto educativo y cultural requiere mentes abiertas a múltiples lógicas en vez de una lógica y considerar una variedad de rutas, puntos de vista y perspectivas inclusivas acordes con la tensión cultural y educativa de la complejidad. La intersubjetividad, en sentido educativo, se basa en una tensión dialogante, plural y abierta a la promoción de procesos de entendimiento intercultural y de interdependencia y con un carácter de reciprocidad (autonomía, interdependencia). La intersubjetividad, como espacio de relación y de diálogo, necesita una postura reflexiva. La narración, como estrategia educativa de tipo reflexivo, es capaz de mejorar el conocimiento (y por lo tanto las competencias y capacidades) para leer y actuar en relación a ese contexto social. Lo específico de la educación es la promoción de los procesos de aprendizaje que conectan reflexión y narración, escritura y meta-cognición a lo largo de un proceso de re-lectura (y re-escritura) de cada historia y cultura, en el más amplio sentido histórico y cultural de la comunidad y de la sociedad. La narración puede volver a configurar la tensión tradición-innovación, conservación-cambio.

\begin{abstract}
The educational and cultural challenge of complexity requires an open mind to multilogical rather than monologic one considering a variety of routes, points of view and perspectives in terms of an inclusive cultural and educational tension to complexity. The educability of intersubjectivity is the dialogical, plural and open tension to promote an intercultural process of understanding and interdependence based on reciprocity (autonomy-interdependence). Intersubjectivity, as relational space and dialogue, needs a reflexive posture. The narrative, thought in an educational device, is able to enhance the awareness (and thus skills and capacity) to read and act the relationship with the
\end{abstract}


environment. The educational specificities which therefore fell in promoting learning processes that hold together reflection and storytelling, writing and meta-cognition along a proofreading and re-writing process of individual histories and cultures in the broadest of their community and society. The narrative can re-configure the traditioninnovation, conservation-change, tension.

Palabras clave: Complejidad, Intersubjetividad, Aprendizaje, Reflexión, Narración

Keywords: Complexity, Intersubjectivity, Learning, Reflection, Storytelling

\section{Complejidad, participación, aprendizaje.}

La complejidad emergente, expresada a través de numerosos aspectos sociales, de políticas, económicas, culturales y educativas en la contemporaneidad, se comprende dentro de una amplia crisis global que está destruyendo modelos decisionales y culturales auto-referenciados, tautológicos, nomológicos como visiones del mundo privilegiadas.

En estos modelos, y en las visiones culturales que los producen (la efervescencia de diferentes prospectivas que muestran diversos actores, las instancias participativas de numerosos puntos de vista); la construcción social de las narraciones múltiples de los sujetos, grupos, comunidades y necesidades que estos últimos expresan, se excluyen sistemáticamente de procesos de producción de sentido y de decisión (Crouch, 2003).

Dichos sujetos y sus instancias, se consideran destinatarios y espectadores de elecciones tomadas desde la lógica del poder (cultural y económico) dominante (Sassen, 1992; Le Galès, 2002).

Al mismo tiempo, a través de la participación, nuevos actores expresan un mapa complejo de necesidades, deseos, saberes locales, competencias que piden legitimación y que no están reconocidos por las formas precarias de representación política (Dahl, 1992; Beck, 2000). El desafío global, participativo e intercultural que exige la complejidad contemporánea, más que una nueva moda ideológica, representa un proyecto y un reto metodológico, y por tanto pedagógico y social, para superar las contradicciones subyacentes con paradigmas culturales antagónicos, a lo largo del continuum local-global (Mander, Goldsmith, 2000). Esto significa concentrar la acción educativa y la reflexión pedagógica sobre la interacción de múltiples visiones en las recíprocas diferencias a lo largo de una constante tensión entre universalización y localismo. En esta dirección la universalización representa el desafío pedagógicoeducativo que puede darse solo a partir de un diálogo intercultural con las "ontologías locales" en el interior de un proceso inclusivo, intercultural, dialógico que "cultiva la intersubjetividad" como pluralidad de valores (Morin, 2000). Al mismo tiempo, la desmenuza en grandes narraciones totalizantes (Lyotard, 1981) que han polarizado el debate cultural y político del último siglo y cercanas a las definiciones de los mitos de hoy de Barthes (1974) y de la doxa de Bourdieu (2003), que hacen referencia a la crisis 
de "sentido común" y del orden social y cultural cristalizado hacia el cual se había dirigido, entre otras, la crítica de Gramsci (1934).

La crisis del sentido común y de la erosión de las hegemonías culturales dominantes (Gruppi, 1972) tiene que ver con la convicción histórica madurada en la tardíamodernidad de que tales narraciones eran estables y definitivas, más que construcciones-constricciones culturales producidas por conflictos de poder a lo largo de la historia del ser humano. El carácter de incertidumbre de las condiciones contemporáneas se debe a la parcialidad cultural y científica de visiones culturales concebidas como antagónicas y recíprocamente auto-suficientes y excluyentes. El termino crisis designa la ruptura de un equilibrio precedentemente constituido e implica la transformación de consuetudinarios esquemas interpretativos y de acción que se revelan inadecuados para los sujetos involucrados en las nuevas exigencias del contexto y de los contenidos de la relación (Morin, 1980, p. 211). En tal sentido, la crisis de la modernidad tiene que ver con la mutada percepción de no poder explicar y actuar sobre la realidad, dentro de un enfoque contrastante y dialectico donde se presuma la presencia de:

- Un punto de vista privilegiado ab interno como referencia sobre una antagónica visión cultural;

- Un punto de vista privilegiado ab esterno, que supere tal dualismo.

La condición postmoderna y la presencia de una "pensamiento débil" exige considerar la necesidad de un diálogo intercultural entre diferentes paradigmas y enfoques, allí donde la complejidad permite una multiplicidad de puntos de vista que no pueden ser sintetizados de manera exclusiva y excluyentes a través de la adopción de una "metapunto de vista" (Morin, 1974).

Nuestra época, aun perteneciente a la modernidad (modernidad tardía) está caracterizada por una dimensión reflexiva de auto-critica (Beck, Giddens, Lash, 1999). El declive de la modernidad y la superación de la misma, pasa de la posibilidad dialógica y plural de encontrar respuestas (y formular preguntas) complejas, plurales e incluyentes y utilizar un lenguaje compartido capaz de encarar la complejidad en los continuos equilibrios que los momentos de incertidumbre provocan entre paradigmas culturales antitéticos (instancias instituidas e instituyentes).

Este choque paradigmático y cultural recuperable en el enfoque tradicional dialéctico, manifiesta hoy día la necesidad dialógica de la co-existencia de perspectivas que trabajan en el cambio social, focalizándose en las interconexiones entre el nivel bottom up y el top-down. De hecho, respeto al estado y a la radicalización del debate entre paradigmas culturales contrapuestos y antagónicos, se generó poco a poco una situación de doble vínculo, que puede producir un esquimogénesis a escala nacional e internacional (Watzlawick, et al, 1967, pp. 99-111).

Tales parálisis comunicativas producen de manera ambivalente procesos de desintegración del sistema instituido, frente a la irrupción de fuerzas instituyentes o el fortalecimiento del sistema instituido frente a un aniquilamiento (disoluciónfagocitación) de las especificidades y de las diferencias de las cuales son portadoras gracias a múltiples instancias emergentes.

De hecho, incapaces de pensar en términos incluyentes (dialógicos más que dialécticos), se tiende a generar un doble vínculo. 
Este doble vínculo comunicativo encuentra su superación siguiendo una opción dialógica y transcultural (vel-vel) basada en el reconocimiento recíproco frente a un proceso dinámico de mutua identificación cultural (Geertz, 1998). En la superación de este vínculo dialéctico entre diferentes posiciones culturales, es posible reconfigurar la relación de coexistencia entre múltiples niveles en un proceso de mutuo reconocimiento, de construcción social de sentido (Morin, 1995, p. 43).

El desafió educativo y cultural de la complejidad impone la adopción de una forma de pensamiento abierta de tipo multilógico, más que monológico, que considera una multiplicidad de caminos, puntos de vistas y perspectivas inclusivas como la tensión cultural y educativa de la complejidad.

En este sentido, el mismo Visalberghi considera la necesidad de promover la educación del pensamiento complejo, donde la acción educativa y la reflexión pedagógica puedan construir un tipo de pensamiento abierto, flexible y por tanto complejo (Visalberghi, 1987). De esta manera, el proceso debe fundamentarse en la comprensión, como un proyecto de convivencia social a co-construir dentro de una ética y de una cultura plural, dialógica, abierta, planetaria (Morin, 2001).

De hecho, como sostienen Maturana y Varela: "Crecer como miembro de una sociedad consiste en volverte estructuralmente parte de ella;" (Maturana, Varela, 1985, p. 42).

Es decir como diría Bateson, una tensión a partir de la necesidad de creer tanto en la acción colectiva como en la inevitable diferencia con la cual el deseo individual se le opone (Bateson, 1976). Los sistemas complejos son auto-eco/organizados, de hecho, en el marco ecológico-sistémico existe un "camino del medio" entre autonomía y heteronimia que permite pensar "la co-emergencia de las unidades autónomas y de sus mundos" (Varela, 1985, p. 132). Desde este punto de vista un sistema es, por definición, auto-poietico, es decir, tiene la capacidad di auto-construirse, quedando abierto a las influencias y perturbaciones del ambiente externo (Maturana, Varela, 1987). Esta continua re-definición del sistema hacia un equilibrio dinámico representa la condición de autonomía de los agentes de la interacción social, la cual se debe precisamente a su "heteronimia" en el sentido de que las constricciones normativas externas puedan ser configuradas como "recursos" (capacidad y competencia) que hagan posible la acción en relación con los otros (Steiner, Stewart, 2009, p. 527).

La crisis percibida como oportunidad, puede representar el punto de comienzo de una relación comunicativa de tipo ecológico-sistémico, como tensión dinámica hacia la coevolución entre diferentes puntos de vista a lo largo de un proceso de deuterioaprendizaje capaz de desarrollar la capacidad de sistemas sociales e culturales de auto/organizarse mejorando su propia autonomía en la interdependencia con otros sistemas a lo largo de una reciprocidad de intercambio comunicativos (Zizek, 2012).

\section{Acción pedagógica como proyecto comunicativo dialógico e intercultural}

Respeto al discurso ya iniciado, nos viene útil la definición de cultura entendida como "telaraña de significados" formulada por Geertz: "Una estructura de significados transmitida históricamente, encarnados en símbolos, un sistema de concepciones hereditarias expresadas con formas simbólicas por medio del cual los seres humanos comunican, perpetúan y desarrollan su conocimiento y sus actitudes delante de la vida" (Geertz, 1987, p.141). Acorde con todo lo explicitado en el párrafo anterior, Maturana y Varela la definieron reciprocidad de comportamientos significativos (Maturana, Varela, 
1987). El orden simbólico dado por la coexistencia en un ambiente común (el mundo de la vida de Husserl) se co-construye a través de prácticas sociales y relaciones que tienen en la base, como afirma también Mead, y luego Vygotskij y Habermas, el lenguaje (Mead, G. H., 1966).

Berger y Luckmann consideran el sentido común "el conocimiento que yo comparto con otros en la normal, auto-evidente rutina de la vida cotidiana" (Berger, P., Luckmann, T., 1979, p. 44). El sentido común, como curso de acción ritual, es la norma (no solo comunicativa) a partir de la cual se forma la cultura dominante. En tal sentido las guerras, afirmaba Wallerstein, son guerras de significados, o sea principalmente de tipo semántico (Wallerstein, I., 2006). Es el mismo mundo que en la interpretación postmoderna emerge como compleja interrelación de "procesos locales en continua lucha y negociación sobre significados, valores y recursos. Todos somos arquitectos de la "modernidad" y "caníbales del orden social" (Bauman, Z., 1999). Esto parece coherente con lo definido por Habermas, el cual, ha enfrentado la cultura respeto al nexo que la une fuertemente al binomio comunicación y poder Habermas (1996). En la contemporaneidad, sin embargo, parece verídico el hecho de que estas reglas estén decididas en la mayoría de las relaciones de poder con el sistema dominante y se fundamenten precisamente en la cristalización del sentido común (a través de una comunicación redundante y recursiva). "Los confines y las jerarquías están siempre establecidos por un observador, cuyas operaciones y decisiones intervienen a distintos niveles en el proceso de construcción de un sistema” (Bocchi, Ceruti, 2007, p. 8). El sentido común al que se hace referencia en el interior de una comunidad de hablantes, es el espacio educativo del cambio cultural y social sobre los cuales Gramsci ponía énfasis con la promoción del sentido crítico. El sentido crítico es un proceso de reflexión sobre la experiencia propia de cada uno, es decir, una narración que es una reconfiguración de sentido (aprender a aprender) capaz de releer y poner en marcha los nexos entre lo vivido individual y colectivamente (Bateson, 1976). Para Habermas (1996), la acción comunicativa se debe pensar como acción intercultural donde la ética de la comunicación considere la intersubjetividad como premisa para construir la comunidad de la comunicación. De todas formas, el "sentido de la realidad" que Bauman considera como un acto de responsabilidad hacia la alteridad y la autonomía en el recíproco reconocimiento del otro, da la importancia de la sociedad con sentido ético y civil.

Como especifica Bauman "asumir la responsabilidad hacia la alteridad, la identidad y la autonomía del otro es una condición crucial para la realización de cada individuo y es parte indispensable de cada auténtica «pragmática del intercambio»" (Bauman, 1999).

La existencia se manifiesta como una continua apertura intersubjetiva, esperando la presencia de la alteridad en el devenir humano. La comunicación de tipo no dialéctico tiende a la esquismogénesis entre instancias centrífugas-centrípetas de heteronimiaautonomía, mientras que la de tipo ecológico-sistémica representa un proyecto educativo para llegar a un abanico de reglas acordadas en una "comunidad hablante": un proyecto intercultural y dialógico que se fundamente en un desafío educativo con el objeto de promover capacidades y competencias que vayan en esta dirección (Le Boterf, 2008).

La existencia tiene que ver entonces con una tensión hacia la autonomía, que se explica según una dimensión dialógica, intercultural, plural, o sea en una condición relacional de intersubjetividad: auto representándose como intencionalidad que se abre a otros 
sujetos. La intersubjetividad como proceso educable según un enfoque ecológicosistémico, puede asumir una función mediadora en cuanto que genera "intercambios simbólicos" de tipo comunicativo entre sujetos que participan a un proceso comunicativo. Estos intercambios simbólicos definen la mediación del mundo, lo cual se realiza a través de lenguaje. La posibilidad de educar a la intersubjetividad como construcción pedagógica representa la tensión dialógica, plural y abierta que promueve un proceso intercultural de comprensión y de interdependencia basado en la reciprocidad. La reciprocidad se entiende como tensión dialógica basada en aspectos de significado en común, sobre los cuales co-construir un proyecto de convivencia cultural de una "ciudadanía planetaria", en términos de reciprocidad de comportamientos significativos basados en el consenso, la intersección, la razón. En el mundo que conjuntamente vivimos con los demás (Lebenswelt), la intersubjetividad, en cuanto a espacio relacional y dialógico, se caracteriza por una postura reflexiva y de observación interna (cibernética de segundo orden) que se vuelve autoconsciente. Esta intersubjetividad está definida por Morin como autorreflexión universal. Para Freire y la pedagogía libertaria, a partir de un proceso de auto-conciencia de la propia condición, se produce una tensión hacia el otro, como posibilidad de diálogo. "El diálogo es ese encuentro de ser humano, a través de la mediación del mundo, para dar un nombre, y entonces no se acaba en la relación yo-tú” (Freire, 2002, p.78). La intersubjetividad como implicación cognitiva y emotiva tiene que ver entonces con "estar en cuanto ser-en el mundo es contemporáneamente un ser-el uno con el otro, un ser con otros" (Heidegger, 2012, p.32). Además, Ricoeur en la obra Si mismo como otro explica la cuestión de la identidad como una alteridad de nivel originario y profundo en el proceso de constitución del sí mismo (Ricoeur, 1993). La pedagogía trabaja con la intersubjetividad y está interesada en la construcción de un sentido crítico y el desarrollo de competencias y capacidades para mejorar la adaptación recíproca entre persona y ambiente (sujetos, grupos, comunidad, institución), a través de un proceso de negociación entre las diversas perspectivas y de co-construcción social finalizada precisamente en el equilibrio dinámico de tipo auto/co-evolutivo. Según la perspectiva sistémico-constructivista, los sujetos atribuyen sentido y valor a la propia experiencia interaccionando con los otros a partir del conjunto de beliefs personales (creencias, valores, actitudes, etc.) que derivan del contexto y de situar dentro la interacción y contribuyen, gracias a un proceso de re-interacción, a la re-definición del mismo contexto y de sus reglas (Garfinkel, 1974, pp. 15-18). En el proceso comunicativo, las representaciones del sujeto, mediadas por el contexto cultural en el que participa (sentido común) y también en las representaciones de los otros, permiten, a través de continuas retroalimentaciones (retroacciones positivas/negativas) dar significado a la acción social, con la finalidad de mantener coherencia en el propio sistema de representaciones y entre él y el comportamiento propio hacia los demás (Peirce, 1994). La comunicación permite no solo el intercambio de informaciones para codificar-recodificar a partir de diferentes sistemas de premisas (beliefs), y se produce también la negociación de significados desde de los diferentes puntos de vista. Este proceso de negociación de significados es un proceso educativo de co-construcción social que contribuye a definir, al mismo tiempo, las identidades individuales y las identidades colectivas. El carácter de imbricación entre el nivel individual y social, esto es el diálogo entre sistemas comunicativos, es definido por Bateson como "the patterns which connect" (Bateson, 1972) y ocurre en dos niveles: la construcción individual y la co-construcción colectiva. En este sentido, la dimensión individual, igualmente 
imbricada en la social, modula instancias de continuidad-cambio que permiten la relación con los otros, retomando estrategias aptas a mantener una coherencia entre premisas y comportamientos a partir de los significados asignados a los comportamientos de uno mismo y de los demás (Goffmann, 1988). El segundo nivel se refiere al proceso de co-construcción de la realidad como proceso social a partir de las interacciones comunicativas que se activan por diferentes sistemas sociales, que son actores de la comunicación. Este segundo nivel está implicado en los procesos de negociación de beliefs (significados, valores, etc.), que los actores realizan a través de intercambios lingüísticos para sintonizarse con los otros co-participantes en la coconstrucción de la realidad. Desde esta óptica, la dimensión educativa tiene como finalidad promover procesos de cambio como actividad situada y emergente de la interacción entre un actor cognitivo y los vínculos contextuales, actividad, distribuida y compartida, a la cual concurren la naturaleza y la historia de las relaciones, entre los individuos implicados, las características físicas del ambiente, las herramientas de la cultura (Liverta Sempio, Marchetti, 1995).

Promover la reflexión de los actores y de sus "mundos" equivale a estimular su capacidad de (auto)diseñarse frente a las paradojas y las ambigüedades de los fenómenos de pérdida de la tradición, de puesta en duda de las certezas racionales y de la estabilidad de los conocimientos.

Dewey de hecho afirma que "aprender a ser humanos significa desarrollar aquel hacer y recibir que constituyen la comunicación” (Dewey, 1971, p.121).

En este sentido, "la pedagogía, que trabaja para que el sujeto se adapte a su ambiente, pero sobre todo para que sea capaz de diseñar proyectos, con los cuales trabajar y adaptar su ambiente a sí mismo, a sus necesidades, sus deseos, su imaginación" (Colazzo, 2011, p.8). Educar al cambio social significa por lo tanto monitorear y facilitar intervenciones educativas dirigidas a generar ocasiones y estímulos para reflexionar sobre el funcionamiento de la sociedad y de sus vínculos, que ella impone, resurgiendo estrategia de autonomía y de acción que sabemos tender entre las instancias de transmisión y transformación social.

La pedagogía, como regulador comunicativo y por su carácter de mediación, tiende como objetivo a realizar el ser humano en la sociedad, llevando a un equilibrio dinámico de convivencia entre las diferencias, esto es considerando como procesos complementarios de cambio y estabilidad. "La pedagogía de la postmodernidad puede trabajar para ser contemporánea en su tiempo, para construir la libertad y la autonomía de los sujetos, favoreciendo sus actividades de decisión y elección, promoviendo una construcción racional abierta a la intersubjetividad, al diálogo, a la discusión, a gusto de la negociación de significados" (Colazzo, 2005, p. 29). En este sentido el proceso de co-construcción del conocimiento y de la cultura se define por su carácter intrínsecamente intersubjetivo y de auto/co-construcción social a partir del contexto cotidiano en el cual se consumen retroalimentación positiva (en sentido de acción) y retroalimentación negativa (ídem) en la búsqueda de continuos equilibrios de los diferentes sistemas sociales y múltiples referencias culturales. En relación a las construcciones de "sentido común gramsciano", de la "Doxa" de Bourdieu, de las "falsas creencias de Pierce" la literatura sistémico-constructivista nos detalla que los procesos de reflexión, aprender a aprender, meta-cognición, representan un desafío educativo para releerse a uno mismo y las relaciones entre uno mismo y el contexto cultural en el que vive. En este sentido, el aprendizaje no hace nada más que promover 
procesos de relectura de los límites individuales y contextuales, detectando estrategias y recursos para actuar la complejidad contemporánea. Archer (2006) subraya la influencia de la cultura sobre las personas y la capacidad de estas de tomar la "conversación interior" precisamente para comprender y actuar socialmente. Esta conversación interior representa una modalidad reflexiva para reelaborar lo que emerge exteriormente, es decir desde la estructura social. El diálogo entre inconsciente/consciente-cognitivo e inconsciente/consciente social representa la posibilidad de adaptarse creativamente a la sociedad teniendo márgenes de libertad y de transformación partiendo precisamente de la reelaboración ab interno (internamente) de los estímulos y de los vínculos expresados social y culturalmente, poniendo en conexión la dimensión tacita e inconsciente de las preferencias y trabajando precisamente sobre el fortalecimiento de esas competencias y capacidades.

La auto-reflexión permite conectar el mundo interior con el mundo exterior a través de un enfoque hermenéutico y fundamentado en el construccionismo social que permite enlazar la red de vivencias individuales y colectivas. De esta manera la narración biográfica permite reconfigurar la identidad como un proceso de co-construcción social. Así, la narración de las personas, de los grupos, de las comunidades se escribe e inscribe en una narración más amplia que es la memoria colectiva (Levy, 1996) y las conexiones sociales (De Kerckhove, 1997).

Recientemente un texto importante como el redactado por Kaneklin y Scaratti (1998), remarca el valor de la narración como instrumento indispensable tanto para la construcción de significados, de prácticas simbólicas como de artefactos materiales e inmateriales (cognitivo-culturales), como para facilitar procesos de cambio social y organizativo en contextos sociales y educativos. La narración, se relaciona con lo experimentado por los sujetos en su atribución de sentido a eventos y a la acción social. La educación se entiende entonces no solo como transmisión de conocimiento, sino como relación entre personas que intercambian experiencias a través de la narración y el intercambio recíproco de información (Mantegazza, 1999).

\section{Reflexiòn, narración, memoria conectiva.}

"Problematizar nuestra cultura, no para destruirla, sino para hacer emerger las posibilidades tácitas durante los siglos a través de un proceso mayéutico" (Freire, 1971, p.11).

Como hemos sintetizado en los parágrafos precedentes, el homo narrans representa una condición histórica localizable ya desde la prehistoria y representa una exigencia relacional de profunda relectura en la propia experiencia cuotidiana de vida dentro contextos culturales mucho más amplios (Benjamin, 1976, p. 235; Eco, 1993). Todo esto aparece mucho más decisivo en la complejidad contemporánea, debido a la ausencia de mitos unitarios y monolíticos a los cuales hacía referencia, entre otros, Barthes y Lyotard respecto a una cultura entendida en manera orgánica y jerárquica. Es decir, a la imposibilidad de dar una representación exhaustiva, totalizadora y tranquilizante de la realidad, que pueda "reducir" la complejidad en la cual vivimos, y que contemple la cultura como narración compartible y estática.

Hall, a este respecto observa que "Las identidades son relativas a las modalidades en las cuales nos posicionan y en las que nos posicionamos en las numerosas narraciones" 
(Hall, 1990, p. 222). El constructo de aprender a aprender, como hemos evidenciado en las páginas precedentes, nos permite releer la propia biografía, memoria y narración de una manera en que son interdependientes con el contexto social y de la comunidad, que se modifica y con el cual somos modificados también nosotros, a través una tensión entre simetría y complementariedad (autonomía-heteronimia). De este modo la narración biográfica nos permite reconfigurar la identidad como un proceso de construcción social en el cual la narración de las personas, de los grupos, y de las comunidades se escribe y circunscribe en una narración más amplia, que es la memoria colectiva y conectiva de tipo social.

Adoptar un dispositivo educativo basado en el nexo memoria-narración permite releer la vivencia de los sujetos y de las comunidades para que podamos encontrar, de este modo, márgenes de redefinición de lo propio, sentidos como una orientación en la sociedad. Como sostiene M. Callari Galli, el pasado cultural y la memoria de las comunidades no debería ser considerado solo como un instrumento para promover la participación de un sujeto en su comunidad sino más bien como un impulso hacia un proceso de co-construcción entendido éste como un nuevo proyecto cultural de la comunidad. De este modo es posible re-configurar fragmentos de la memoria del pasado conectando las generaciones futuras en un proyecto de participación y de nueva ciudadanía en la vida de la comunidad (Callari Galli, et al, 1998, pp. 191-192). La oportunidad de salida de la vinculación que impone la cultura-memoria dominante, hace posible la re-configuración, dentro de un proceso educativo, de un sentido intercultural e intersubjetivo compartido por los sujetos de las comunidades; y en el sentido más amplio, por la sociedad, pensado éste como un espacio de construcción socio-cultural de convivencia civil. La narración individual y grupal, como proceso reflexivo de deutero-aprendizaje re-configura la tensión que existe entre tradicióninnovación, conservación-cambio. Retomando a Bateson en su contribución con el concepto de deutero-aprendizaje, el proceso de re-memorización nos va a permitir una definición de las propias coordinadas existenciales (en el presente-pasado-futuro), a través de la crisis que propone una re-configuración de los complejos equilibrios que encontramos entre conservación-mutación y entre persona-comunidad-sociedad (individuo-ambiente). En este sentido, en uno de sus trabajos más significativos, Giddens afirma que las estructuras sociales son de hecho huellas de la memoria localizables en las prácticas cuotidianas (Giddens, 1990).

La memoria colectiva y conectiva de las comunidades representa una narración mediada y socialmente construida por las relaciones e interacciones de las memorias biográficas de los sujetos. Además, la perspectiva funcionalista de la memoria colectiva propuesta por Halbawchs tiende a subrayar su carácter estático y de continuidad, asumiendo ésta una connotación abductiva, es decir hecha, de continuas re-definiciones entre las memorias individuales emergentes y las fijadas por la memoria instituida (sentido común). Narrar "el nosotros" es un modo para releerse y para releernos entre los vínculos y las posibilidades, encontrando de este modo un camino practicable hacia una autonomía interdependiente en el más amplio contexto cultural y social posible. Por consiguiente, narrar es actuar, dado que, en estos términos el narrar nunca describe, sino que actúa sobre mi relato, sobre el otro escuchado (Geertz, 1987). Jedlowski, recorriendo los nudos epistemológicos de la sociología de la vida cuotidiana se centra en la importancia de esta como espacio de reflexión, de narración y de re-construcción social de la propia identidad dentro de un grupo, comunidad, sociedad. Para promover 
un cambio en la sociedad es necesario partir de los artefactos culturales que producen el sentido común o lo que es lo mismo, ese que es más próximo: la memoria colectiva. Es decir, una consciencia colectiva de las interdependencias entre las memorias biográficas individuales y la conectiva de las comunidades y de la sociedad que, más que ser un anclaje nostálgico del pasado se convierte en un impulso proponente para proyectar horizontes de sentido compartido. La condición de atravesar dichos horizontes como característica dialógica de las identidades contemporáneas es expresada también en ámbito antropológico por parte de autores como Clifford e Geertz. Ambos consideran la cuestión antropológica contemporánea como cuestión postcolonial a partir de la consideración de una cultura trans-local que expresa la identidad entendida como "travelling cultures" y que reflexiona sobre las propias prácticas de atribución de sentido "writing cultures" (Clifford, 1999, p. 19). Tales puntos de vista, después de continuos ajustes entre las exigencias de conservacióncambio/ tradición-innovación, reconfiguran equilibrios nuevos mucho más complejos.

Este proceso de negociación entre memorias, representaciones, vivencias individuales y memorias colectivas se constituye como una tensión proactiva hacia el cambio que encuentra en la aportación teórico- metodológica de la narración, un dispositivo pedagógico, una modalidad eficaz para supervisar y facilitar prácticas interculturales entre personas-grupos-comunidades-sociedades. Del mismo modo el método narrativo favorece un proceso educativo de auto-reflexión, de auto-concienciación y de autoproyección en una tensión entre conservación-transformación/ tradición-innovación/ exigencias y representaciones individuales y colectivas. Por lo tanto, la narración representa un proceso de reconfiguración identitaria de la propia historia personal, y es producto y productora de una historia comunitaria y social. La reflexión, activa un proceso reflexivo de relectura de la experiencia (experiencia de segundo nivel) propio del enfoque sistémico-constructivista. A través de la narración, entendida de hecho, como reflexión sobre la experiencia, nos releemos a nosotros mismos y re-escribimos el sentido de nuestros hechos cotidianos (Jedlowski, 2000). En este sentido hay una diferencia sustancial entre hacer experiencia y tener experiencia. En el segundo caso está claro que el reflexionar es también contarse, es decir, la posibilidad de no limitarse al hecho en sí mismo, sino que se le dé otro significado accesible también a otros mediante la utilización de la escritura (Jedlowski, 1986). De hecho, el hombre actual tiene sed de narración porque en la narración re-encuentra espacio y tiempo para la propia vida (Knowles, 1996). Desde el punto de vista de la valoración educativa de la narración, como sostiene Bruner (1993), el pensamiento narrativo se ocupa del particular, de las intenciones y de las acciones del hombre, de las vicisitudes y de los resultados. Su intención es la de situar la experiencia en el tiempo y en el espacio.

El trabajo educativo se entiende por tanto en cuanto que promueve el desarrollo de la inteligencia narrativa para que pueda ser inteligencia hermenéutica, como capacidad que va a la búsqueda del significado y de los demás; es decir consciencia de nosotros mismos en relación con los demás (Gardner, 2002). "El mundo de la vida cotidiana es la escena y también el objeto de nuestras acciones e interacciones. Tenemos que dominarlo y cambiarlo para llevar a cabo las finalidades que perseguimos en ella entre nuestros símiles. (....) El mundo en este sentido es algo que tenemos que modificar mediante nuestras acciones y que a su vez modifica también las nuestras" (Schutz, 1975, p.13). 
La especificidad de lo educativo se refiere por tanto a la promoción de procesos de aprendizaje que concilien reflexión y narración, escritura y meta-conocimiento a través un proceso de relectura (y re-escritura) de la propia historia y de la propia cultura y concretamente en la propia comunidad y por consiguiente en la sociedad (Jedlowski, 1994). En este sentido la memoria misma entendida como un proceso selectivo representa el perno sobre el cual se construye la identidad entre pasado, presente y futuro en una mediación, entre la memoria individual y colectiva, así como entre la exigencia presente entre transmisión/reproducción y entre re-interpretación y redefinición de la realidad. Este mapeo de las formas de narración nos hace conscientes de que el discurso se mueve en la dinámica yo-otro y no puede omitir su naturaleza relacional. La identidad cotidiana es tal en cuanto que, está en relación a través de una implicación de prácticas, Es decir, uno se percibe como sujeto, como persona que en, desde y sobre la comunidad se narra. Outsider inside, propone Collins, además de las falsas alternativas paradójicas de la doble vinculación desveladas por Watzlawick (Collins, 1986).

El objetivo de esta relación es el de incidir, haciendo visible este confín negociado que se lleva a cabo con los demás, la definición de una narración conectada. En este sentido, el conocimiento no es un sistema jerárquico sino un sistema de co-construcción y de interconexiones que garantiza la continua evolución y re-configuración del saber. La narración puede potenciar una mejor concienciación de las relaciones con el ambiente, de la singularidad de la propia pertenencia incluyendo la propia biografía en un universo de sentido más amplio. La narración como instrumento de concienciación de las memorias y de las biografías individuales permite una re-lectura de las experiencias, de las tradiciones, de las biografías, de los relatos populares, de los conocimientos tácitos, de los saberes locales, en cuanto que la identidad es un proceso circular de negociación y de co-construcción social entre las diferentes personas que viven en una comunidad. Por lo tanto, es un proceso lento de estratificación, sincrético y de equilibrios contemporáneos entre tradición-innovación, continuidadtransformación. Como sostiene Colom, la narración, sea como proceso individual o colectivo, permite que nuestra percepción sea participada y compartida con los demás traduciéndose en artefacto simbólicos y en prácticas que se convierten de este modo en un metáfora de la realidad, es decir una construcciòn social de la realidad (Colom, 2002).

\section{Referencias Bibliográficas}

Archer, M. S. (2006). La conversazione interiore. Come nasce l'agire sociale. Torino: Centro Studi Erickson.

Barthes, R. (1974). I miti d'oggi. Torino: Einaudi.

Bateson, G. (1972). Mente e natura. Milano: Adelphi.

Bateson, G. (1979). Verso una ecologia della mente. Milano: Adelphi.

Bauman, Z. (1999). La società dell'incertezza. Bologna: Il Mulino.

Beck, U., IN Beck, U., Giddens, A., Lash, S. (1999). Modernizzazione riflessiva. Trieste: Asterios.

Beck, U. (2000). La società del rischio. Roma: Carocci.

Benjamin, W. (1976). Considerazioni sull'opera di Nicola Leskov, in Angelus Novus. Torino: Einaudi. 
Berger, P., Luckmann, T. (1969). La realtà come costruzione sociale. Bologna: Il Mulino.

Bocchi, G., Ceruti, M. (2007). La sfida della complessità. Milano: Feltrinelli.

Bourdieu, P. (2003). Per una teoria della pratica con tre studi di etnologia cabila. Milano: Ed. Cortina.

Bruner, J. (1993). La mente a più dimensioni. Bari-Roma: Laterza.

Callari Galli, M., Pievani, T., Ceruti, M. (1998). Pensare la diversità. Per un'educazione alla complessità umana. Roma: Meltemi.

Clifford, J. (1999). Strade. Viaggio e traduzione alla fine del secolo XX. Torino: Bollati Boringhieri.

Colazzo, S. (2011). La pedagogia alla luce del paradigma ecologico-sistemico. Relazione tenuta al Convegno organizzato dalla SIPNEI (Società Italiana di Psiconeuroendocrinoimmunologia), Roma.

Colazzo, S. (2005). Insegnare ed apprendere in rete. Castrignano dei Greci: Amaltea.

Collins, P. H. (1986). Learning from the outsider within. Social Problems, n. 33, 514 532.

Colom, J. A. (2002). La (de)construcción del conocimiento pedagógico: nuevas perspectivas en teoría de la educación. Barcelona: Paidós.

Crouch, C. (2003). Postdemocrazia. Bari-Roma: Laterza.

Dahl, R. (1992). La democracia y sus críticos. Barcelona: Paidós.

De Kerckhove, D. (1997). Intelligenza connettiva. Avvento della web society. Roma: De Laurentis.

Dewey, J. (1971). Comunità e potere. Firenze: La Nuova Italia.

Eco, U. (1993). Sei passeggiate nei boschi narrativi. Milano: Bompiani.

Freire, P. (2002). La pedagogia degli oppressi, Torino: EGA.

Gardner, H. (2002). Formae mentis. Saggio sulla pluralita` dell'intelligenza. Milano: Feltrinelli.

Garfinkel, H. (1974). The origin of the term ethnomethodology, in Ethnomethodology (eds) Turner, R. Harmondsworth.

Geertz, C. (1987). Interpretazione di culture. Bologna: Il Mulino.

Giddens, A. (1990). La costituzione della società. Milano: Edizioni Comunità.

Goffman, E. (1988). Il rituale dell'interazione. Bologna: Il Mulino.

Gramsci, A. (2007). Quaderni del carcere, (eds) Garretana, V. Torino: Einaudi.

Gruppi, L. (1972). Il concetto di egemonia in Gramsci. Roma: Editori Riuniti.

Habermas, J. (1996). Between Facts and Norms: Contributions to a Discourse Theory of Law and Democracy. Cambridge MA: MIT Press.

Hall, S. (1990). Cultural identity and Diaspora, in Rutherford, J., Identity: Community, Culture and Difference. London: Lawrence and Wishart.

Heidegger, M. (2010). Il concetto di tempo. Milano: Adelphi Edizioni.

Jedlowski, P. (2003). I fogli nella valigia. Sociologia e cultura. Bologna: Il Mulino.

Jedlowski, P. (1994). Il sapere dell'esperienza. Milano: Il Saggiatore.

Jedlowski, P. (1986). Il tempo dell'esperienza. Milano: Franco Angeli.

Jedlowski, P. (2000). Storie comuni. La narrazione nella vita quotidiana. Milano: Bruno Mondadori.

Kaneklin, C. Scaratti, G. (eds). (1998). Formazione e narrazione. Milano: Cortina.

Knowles, S. (1996). La formazione degli adulti come autobiografia. Milano: Cortina.

Le Boterf, G. (2008). Costruire le competenze individuali e collettive. Napoli: Guida. 
Le Galès, P. (1992). European Cities: Social Conflicts and Governance. Cornwall: Oxford University Press.

Levy, P. (1996). L’intelligenza collettiva. Per un'antropologia del cyberspazio. Milano: Feltrinelli.

Liverta Sempio, O., Marchetti, A. (eds.) (1995). Il pensiero dell'altro. Contesto, conoscenza e teorie della mente. Milano: Raffaello Cortina.

Lyotard, J. F. (1981). La condizione postmoderna. Milano: Feltrinelli.

Mander, J., Goldsmith, E. (2000). Glocalismo, L'alternativa strategica alla globalizzazione. Bologna: Arianna.

Mantegazza, R. (1999). Un Tempo per narrare. Esperienze di narrazione a scuola e fuori. Torino: EMI.

Maturana, H. R., Varela, F. J. (1987). L'albero della conoscenza. Milano: Garzanti.

Maturana, H., Varela, F. J. (1985). Autopoiesi e cognizione. Venezia: Marsilio.

Mead, G. H. (1966). Mente, sé e società. Firenze: Giunti e Barbera.

Morin, E. (2001). I sette saperi necessari all'educazione del futuro. Milano: R. Cortina.

Morin, E. (1974). Il paradigma perduto. Milano: Bompiani.

Morin, E. (1995). Introduzione al pensiero complesso. Milano: Sperling \& Kupfer.

Morin, E. (2000). La testa ben fatta. Riforma dell'insegnamento e riforma del pensiero. Milano: Cortina.

Morin, E. (1980). Per una teoria della crisi, in D'Eramo, M., (eds). (1980). La crisi del concetto di crisi. Roma: Lerici.

Peirce, B. (1994). Interpersonal communication: making social worlds. New York: Harper Collins.

Ricoeur, P. (1993). Sé come un altro. Milano: JacaBook.

Sassen, S. (1992). The Global City, Princeton. New York: Princeton University Press.

Schutz, A. (1975). Il problema della rilevanza, Riconda, G., (eds). Torino: Rosenberg and Sellier.

Steiner, P., Stewart, J. (2009). From autonomy to heteronomy (and back): the enaction of social life, Phenomenology and the Cognitive Science, 8, 527-550.

Varela, F. (1985). Complessità del cervello e autonomia del vivente, in: Bocchi, G., Ceruti, M. (eds). La sfida della complessità. Milano: Feltrinelli.

Visalberghi, A. (1987). Educare alla complessità del reale, in Scuola e città, 38.

Wallerstein, I. (2006). Comprendere il mondo. Introduzione all'analisi dei sistemamondo. Trieste: Asterios editore.

Watzlawick, P., Beavin, J.H., Jackson D.D. (1967). Pragmatica della comunicazione umana. Roma: AstrolabioRoma.

Zizek, S. (2012). Benvenuti in tempi interessanti. Milano: Ponte delle Grazie. 